\title{
Total coronagraphic extinction of rectangular apertures using linear prolate apodizations
}

\author{
C. Aime, R. Soummer, and A. Ferrari \\ UMR 6525 Astrophysique, Faculté des Sciences, Université de Nice Sophia Antipolis, Parc Valrose, \\ 06108 Nice Cedex 2, France \\ e-mail: Claude.Aime@unice.fr, Andrea.Ferrari@unice.fr
}

Received 25 July 2001 / Accepted 8 March 2002

\begin{abstract}
This paper presents a theoretical study of stellar coronagraphy with apodized entrance apertures. The study is restricted to a perfect telescope operating in space, and a monochromatic on-axis unresolved star. It is shown that linear prolate functions are the optimal apodizers for rectangular apertures in stellar coronagraphy. With the phase mask technique (Roddier \& Roddier 1997), prolate functions can produce a total extinction of the star light. For Lyot's coronagraphy, the extinction is not complete, but prolate apodizations lead to an optimal star residual intensity with surprising interesting properties: the residual star light and the planet enjoy the same apodized intensity pattern (but different dynamic) with the optimal light concentration. With this technique, very high rejection rates can be obtained for Lyot's coronagraphy, with smaller mask sizes.
\end{abstract}

Key words. instrumentation: high angular resolution - technique: high angular resolution - stars: planetary systems

\section{Introduction}

Coronagraphy was first invented by Lyot $(1930,1939)$ for solar corona observations without eclipses. The interest in Stellar Coronagraphy arose later, for the study of the vicinity of stars (Bonneau et al. 1975; Smith \& Terrile 1984; Malbet 1996; Mouillet 1997; Beuzit et al. 1997; Mouillet et al. 1997). Nowadays, coronagraphy is mainly studied for the direct detection of exoplanets, which is principally limited by the intensity ratio between the star and the planet, roughly of $10^{6}$ in the infrared, and up to $10^{9}$ in the visible. The aim of stellar coronagraphy is to remove, as best is possible, the star diffracted light at the position of the planet and several variants of the technique have been studied up to now (Gay \& Rabbia 1996; Roddier \& Roddier 1997; Baudoz et al. 2000a,b; Rouan et al. 2000; Abe et al. 2001).

It has been recently demonstrated (Abe et al. 2002) that the four quadrant coronagraph can perform a total extinction of a monochromatic unresolved on-axis star, for a perfect circular aperture operated in space. We show in this paper that a total extinction can similarly be obtained for Phase Mask coronagraphy, using an entrance apodized aperture.

Send offprint requests to: R. Soummer, e-mail: Remi.Soummer@unice.fr
Lyot's stellar coronagraphy consists of setting a small opaque mask at the center of the star image. The diffraction pattern of this mask appears in negative amplitude on an image of the entrance pupil. There, a diaphragm (Lyot's stop) is set to remove the light diffracted outside the telescope aperture image. Inside this diaphragm, direct and diffracted light interfere destructively. This technique was improved by Roddier \& Roddier (1997), replacing the opaque mask by a $\pi$ phase mask. The efficiency of the destructive interference is about twice as good as for the classical Lyot technique, but the technique is more difficult to implement. For the two techniques (Lyot and Roddier), most of the residual light appears at the edges of the exit pupil, and the extinction is not total, even for a perfect instrument. A well-known empirical solution is to use a Lyot's stop smaller than the pupil.

This pupil reduction can be avoided by the use of an apodized entrance pupil, as proposed independently by Guyon \& Roddier (2000) and Baudoz (1999). Guyon \& Roddier (2000) shown numerically that a reduction factor of up to $10^{7}$ could be obtained with a circular aperture. We recently described similar reduction factors by means of interferometric apodizations for rectangular apertures (Aime et al. 2001a).

In the present paper, we further analyse in depth some theoretical aspects of the association of an entrance 
apodized aperture with a coronagraph for rectangular apertures (a future paper is in preparation for circular apertures). The analysis, which is fully analytic, is restricted to the case of a perfect instrument operated in space, for an on-axis unresolved star. The phase mask is assumed to give a perfect $\pi$ phase shift and the analysis is monochromatic. We show that the optimal theoretical apodizations for coronagraphy (Lyot and Roddier) are given by the linear prolate spheroidal set of functions. A total extinction of the star can be obtained for the Phase Mask. For Lyot's coronagraphy, the theoretical extinction is not total but the prolate apodization provides the optimal star residual light in terms of light concentration.

The paper is organized as follows: Sect. 2 gives the general formalism for Lyot's and Roddier \& Roddier's coronagraphy with entrance pupil apodization. In Sect. 3, for these techniques we analyse the possible prolate solutions of the coronagraphic equation for rectangular apertures. In Sect. 4, we give an alternative presentation of the particular interest of prolate functions for coronagraphy. A conclusion and discussions are given in Sect. 5 .

A few mathematical properties of the prolate functions are summarized in Appendix A. In Appendix B, we compare by numerical simulations the exact prolate solutions to the cosine and cosine squared apodizations we proposed in a recent paper (Aime et al. 2001a).

\section{General equation for coronagraphy with entrance pupil apodization}

In this section, we give the general formalism to describe a coronagraphic experiment with an apodized aperture. Following the presentation of Roddier \& Roddier (1997), the technique involves four successive planes, denoted A (telescope pupil), B (telescope focus, coronagraphic mask), C (relay pupil plane, Lyot's stop) and D (final focus plane).

The complex amplitude at the entrance apodized pupil is proportional to:

$\Psi_{\mathrm{A}}(x, y)=\mathrm{P}(x, y) \Phi(x, y)$

where $\mathrm{P}(x, y)$ is a function of transmission 1 inside the telescope aperture and 0 outside and $\Phi(x, y)$ denotes the real, positive apodization function, with $\Phi(x, y) \leq 1$.

The wave propagation from the aperture (plane A) to the focal plane (plane B) writes as a scaled Fourier Transform (FT) and we assume that the optical layout is properly designed to eliminate the quadratic phase terms associated with the propagation of the waves (Goodman 1996). In plane B, the coronagraphic mask is applied. Following the notations of Aime et al. (2001b), we have:

$\Psi_{\mathrm{B}}(x, y)=\frac{1}{\imath \lambda f} \widehat{\Psi}_{\mathrm{A}}\left(\frac{x}{\lambda f}, \frac{y}{\lambda f}\right) \times[1-\varepsilon \mathrm{M}(x, y)]$,

where the symbol ${ }^{\wedge}$ denotes the FT, $\lambda$ the wavelength, and $f$ the telescope focal length. $\mathrm{M}(x, y)$ is a function equal to 1 inside the coronagraphic mask and 0 outside that describes the mask shape. We do not consider in this paper other mask functions for which the mask transmission itself is also apodized (Watson et al. 1991).

The parameter $\varepsilon$ plays a fundamental role in coronagraphy; it has only two relevant values: $\varepsilon=1$ for an opaque mask (Lyot's coronagraphy) and $\varepsilon=2$ for a Phase Mask (PM) (Roddier and Roddier's coronagraphy). Other values of $\varepsilon$ might be considered but with less efficiency. Values of $\varepsilon$ lower than 1 would correspond to a partially transparent mask without phase shift (lower efficiency than Lyot's mask), values $1<\varepsilon<2$ to a partially absorbing $\pi$ phase mask (lower efficiency than PM). Values of $\varepsilon$ greater than 2 might also be obtained considering a $\pi$ phase mask surrounded by a partially absorbing screen, therefore reducing the light level of the planet. So, in what follows, $\varepsilon$ only takes the values 1 and 2 .

An optical system is then used to obtain an image of the telescope aperture in plane C. Here also, the complex amplitude of the wave is written using a scaled FT of Eq. (2). For simplicity, we assume that the focal lengths of the successive optical systems are identical (if not, an appropriate change of variables leads to a similar result). Finally, a diaphragm (Lyot's stop), exactly equal to the entrance pupil shape $\mathrm{P}(-x,-y)$, is set in plane $\mathrm{C}$ :

$$
\begin{aligned}
\Psi_{\mathrm{C}}(x, y)= & -\mathrm{P}(-x,-y) \times\left[\Psi_{\mathrm{A}}(-x,-y)\right. \\
& \left.-\frac{\varepsilon}{\lambda^{2} f^{2}} \Psi_{\mathrm{A}}(-x,-y) * \widehat{\mathrm{M}}\left(\frac{x}{\lambda f}, \frac{y}{\lambda f}\right)\right] .
\end{aligned}
$$

A total coronographic extinction for a monochromatic onaxis point source can be obtained if we have: $\Psi_{\mathrm{C}}(x, y)=0$ inside the aperture image. Under this condition, the light coming from the star is entirely diffracted outside the aperture in plane C. Replacing Eq. (1) in Eq. (3), orientating $x$ and $y$ in the direction of $-x$ and $-y$, and using the property $\mathrm{P}(x, y)^{2}=\mathrm{P}(x, y)$ (for a perfect transparent aperture), the condition $\Psi_{\mathrm{C}}(x, y)=0$, may be rewritten as:

$$
\begin{aligned}
\mathrm{P}(x, y)\left[\mathrm{P}(x, y) \Phi(x, y) * \frac{1}{\lambda^{2} f^{2}} \widehat{\mathrm{M}}\left(\frac{-x}{\lambda f}, \frac{-y}{\lambda f}\right)\right]= \\
\frac{1}{\varepsilon} \mathrm{P}(x, y) \Phi(x, y) .
\end{aligned}
$$

We can remove the term $\mathrm{P}(x, y)$ from the two sides of Eq. (4); in that case, we search for the analytic functions $\Phi(x, y)$, solutions to the condition of total coronagraphic extinction:

$\mathrm{P}(x, y) \Phi(x, y) * \frac{1}{\lambda^{2} f^{2}} \widehat{\mathrm{M}}\left(\frac{x}{\lambda f}, \frac{y}{\lambda f}\right)=\frac{1}{\varepsilon} \Phi(x, y)$.

This equation does not admit general solutions for any pupil shape. In this paper, we examine possible solutions, for the particular case of a rectangular aperture. Besides the greater simplicity of the rectangular problem compared to the circular problem, linear apertures provide several advantages (resolution, achromatization) that have already been discussed (Aime et al. 2001b,a) for application to TPF (Terrestrial Planet Finder) or precursor. 

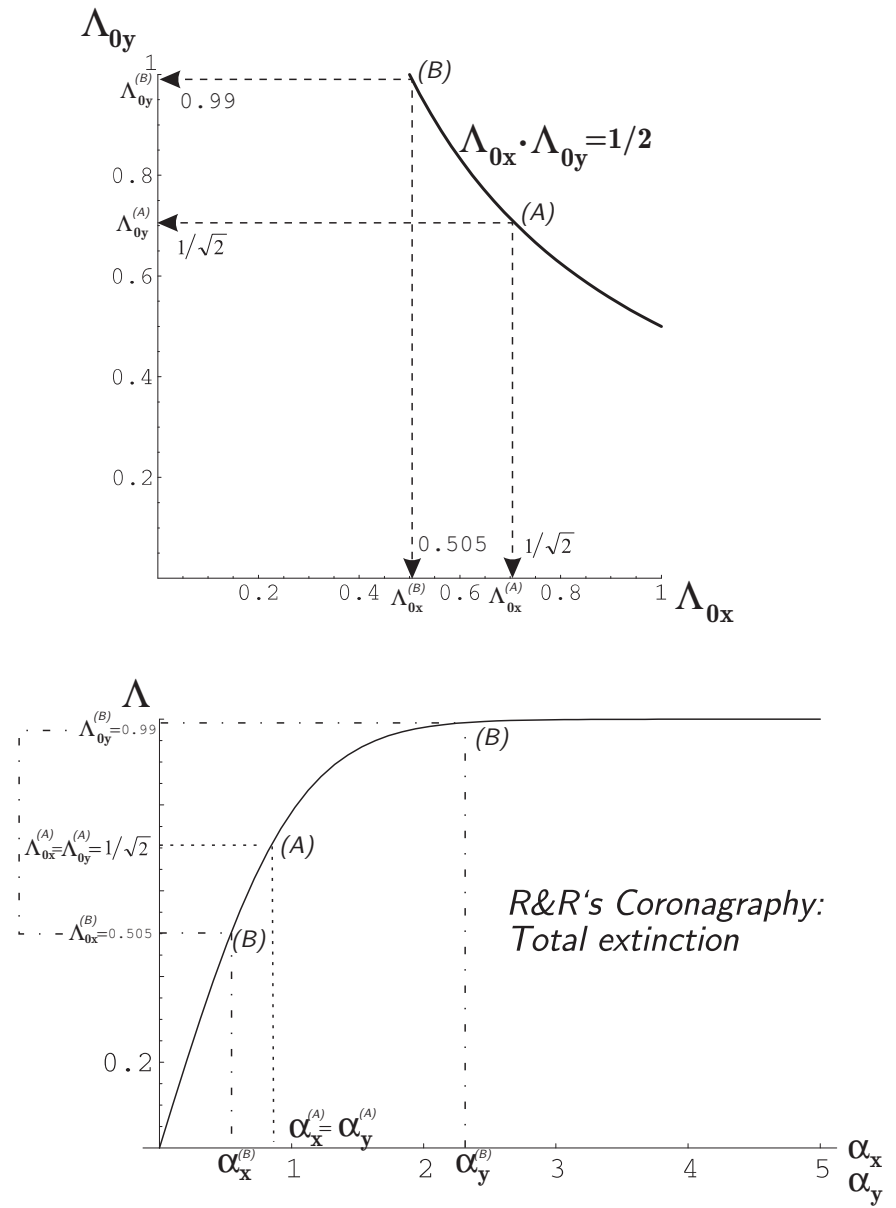

Fig. 1. Top: illustration of the couples of eigenvalues $\left(\Lambda_{0 x}, \Lambda_{0 y}\right)$, solutions to Roddier \& Roddier's coronagraphy. The relation $\Lambda_{0 x} \times \Lambda_{0 y}=1 / 2$ gives an infinity of solutions. The symmetrical solution $(A), \Lambda_{0 x}=\Lambda_{0 y}=1 / \sqrt{2}$ corresponds to the highest pupil transmission (70.6\%). Other eigenvalues may be chosen to reduce the mask size in one direction, such as $(B) \Lambda_{0 x}=0.505$ and $\Lambda_{0 y}=0.99$. Bottom: Eigenvalue $\Lambda_{0 x}$ (or $\Lambda_{0 y}$ ) as a function of the mask size $\alpha_{x}$ or $\alpha_{y}$ (in resolution units). Solution $(B)$ presents the advantage of a smaller mask size in one direction (here $\alpha_{x}^{(B)}=0.547$ ) than the symmetrical solution $\alpha_{x}^{(A)}=0.848$. The transverse mask size is then $\alpha_{y}^{(B)}=2.23$.

\section{Solutions of the general coronagraphic equation for rectangular apertures}

We consider a rectangular aperture of length $L_{x}$ and width $L_{y}$. We assume the apodization function $\Phi(x, y)$ to be separable with respect to the variables $x$ and $y$. The coronagraphic mask $\mathrm{M}(x, y)$ must be a rectangle of size $m_{x} \times m_{y}$ proportional to the diffraction pattern of the rectangular aperture. At the wavelength $\lambda, m_{x}=\alpha_{x} \frac{\lambda f}{L_{x}}$ and $m_{y}=\alpha_{y} \frac{\lambda f}{L_{y}}$, where $\alpha_{x}$ and $\alpha_{y}$ are parameters that express the width of the mask in resolution units. For this rectangular configuration, we have:

$\mathrm{P}(x, y)=\Pi\left(\frac{x}{L_{x}}\right) \Pi\left(\frac{y}{L_{y}}\right)$,

$\Phi(x, y)=\Phi_{x}(x) \Phi_{y}(y)$,

$\mathrm{M}(x, y)=\Pi\left(\frac{x}{m_{x}}\right) \Pi\left(\frac{y}{m_{y}}\right)$,

where $\Pi(x)=1$ for $|x| \leq 1 / 2$ and 0 otherwise.

Substituting the terms of Eq. (6) in Eq. (5), the convolution product separates into two convolution products for $x$ and $y$. The condition of total extinction for rectangular apertures becomes:

$$
\begin{aligned}
{\left[\Pi\left(\frac{x}{L_{x}}\right) \Phi_{x}(x) * \frac{\sin \pi\left(\alpha_{x} / L_{x}\right) x}{\pi x}\right] } \\
\times\left[\Pi\left(\frac{y}{L_{y}}\right) \Phi_{y}(y) * \frac{\sin \pi\left(\alpha_{y} / L_{y}\right) y}{\pi y}\right]= \\
\frac{1}{\varepsilon} \Phi_{x}(x) \Phi_{y}(y) .
\end{aligned}
$$

This integral equation (7) separates into two symmetric requirements, as already described by Frieden (1971) for the study of a confocal laser with square endplates:

$$
\begin{aligned}
& \int_{-L_{x} / 2}^{L_{x} / 2} \Phi_{x}(\xi) \frac{\sin \pi \alpha_{x}(x-\xi) / L_{x}}{\pi(x-\xi)} \mathrm{d} \xi=\frac{1}{\varepsilon_{x}} \Phi_{x}(x) \\
& \int_{-L_{y} / 2}^{L_{y} / 2} \Phi_{y}(\xi) \frac{\sin \pi \alpha_{y}(y-\xi) / L_{y}}{\pi(y-\xi)} \mathrm{d} \xi=\frac{1}{\varepsilon_{y}} \Phi_{y}(y)
\end{aligned}
$$

where $\varepsilon=\varepsilon_{x} \times \varepsilon_{y}$.

For $1 / \varepsilon_{x}$ and $1 / \varepsilon_{y}$ lower than 1 , solutions $\Phi_{x}(x)$ and $\Phi_{y}(y)$ to these relations exist, i.e. a total extinction of the star is obtained. These functions are proportional to the linear prolate spheroidal functions discovered by Slepian \& Pollak (1961). We give a brief presentation of them in Appendix A.

Comparing Eq. (8) and Eq. (A.2), we see that the terms $1 / \varepsilon_{x}$ and $1 / \varepsilon_{y}$ must be identified with the eigenvalues $\Lambda_{n}$ of the prolate functions (positive numbers between 0 and 1). The apodization functions $\Phi_{x}$ and $\Phi_{y}$ must be identified with the prolate functions $\Theta_{n}$ : any prolate function of any order $n$ can be a solution, but we will only be interested in the normalized zero order prolate functions: $\Phi_{x}(x)=\frac{\Theta_{0 x}(x)}{\Theta_{0 x}(0)}$ because it is the only one that is maximum at the origin (maximum transmission at the center of the pupil). This function has already found other interesting application in Optics, as detailed by Frieden in his review paper (Frieden 1971). The two equations of Eq. (8) must be satisfied simultaneously. This means that we can choose any set of eigenvalues $\Lambda_{0 x}, \Lambda_{0 y}$ satisfying the relation $\Lambda_{0 x} \times \Lambda_{0 y}=1 / \varepsilon$. Then, a set of prolate apodizations $\Phi_{x}(x)$ and $\Phi_{y}(y)$ exists (eigenfunctions), with their corresponding mask sizes. 

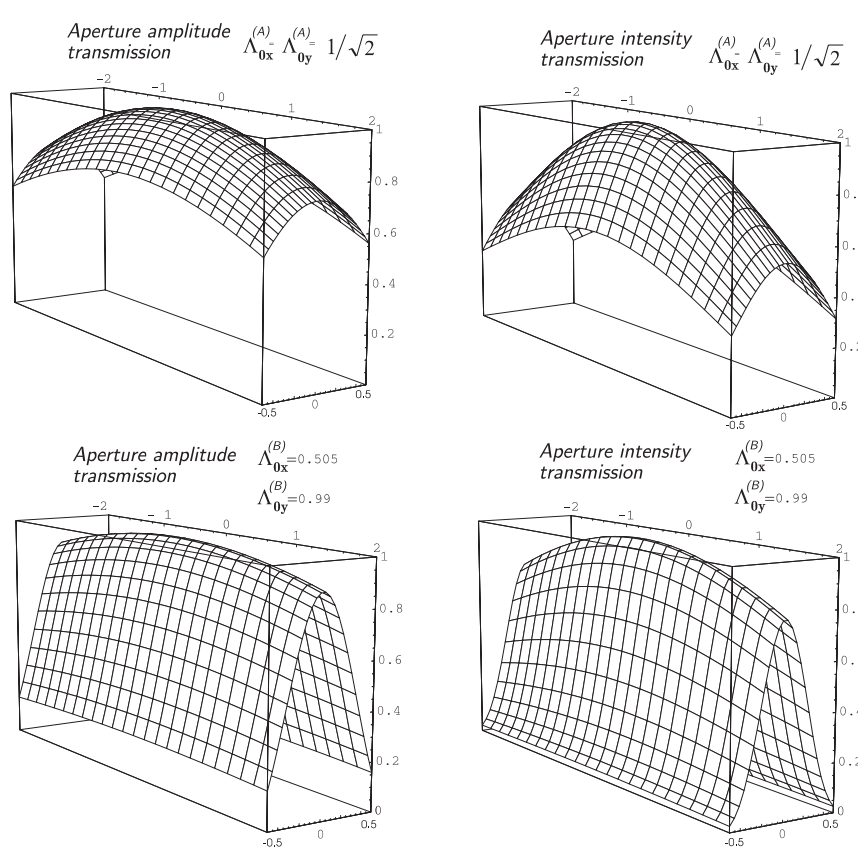
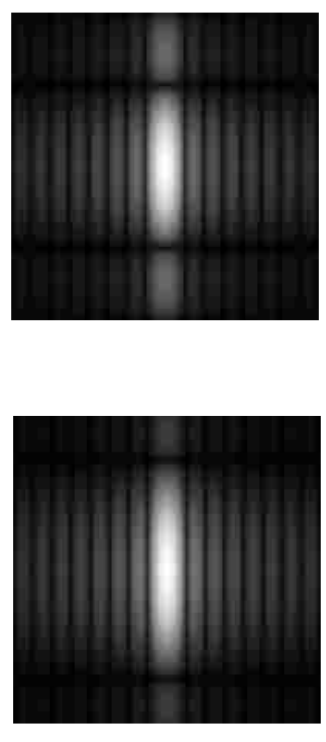

Fig. 2. Examples of entrance pupil apodization (rectangular aperture with $L_{x}=4 L_{y}$ ) for Roddier \& Roddier's coronagraphy that permit a total rejection of the star image. The amplitude and intensity transmissions are drawn for the set $(A)$ of eigenvalues $\Lambda_{0 x}=\Lambda_{0 y}=\frac{1}{\sqrt{2}}$ (top) and for the set $(B) \Lambda_{0 x}=0.99, \Lambda_{0 y}=0.505$ (bottom) of Fig. 1. The overall integrated intensity transmission of the apodizers are: $70.6 \%$ and $47.4 \%$ respectively. Right images: corresponding intensity (non linear scale) in the focal plane where the coronagraphic mask is to be used. In these examples, the apodization effect is mainly visible in the transverse direction.

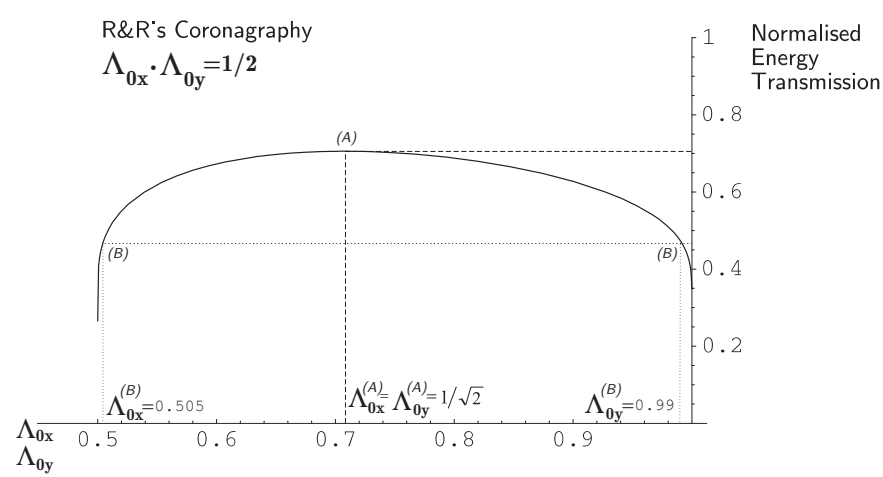

Fig. 3. Energy transmission of the apodized pupil solution to Roddier \& Roddier's coronagraphy (the transmission does not depend on the proportions of the rectangular aperture). The highest transmission $(70.6 \%)$ is obtained for the symmetrical solution $(A) \Lambda_{0 x}=\Lambda_{0 y}=\frac{1}{\sqrt{2}}$. The other configuration $(B)$ of Fig. 1 is also given and its transmission is of $47.4 \%$.

\subsection{Linear prolate solutions for Roddier \& Roddier's coronagraphy $(\varepsilon=2)$}

For Roddier \& Roddier's coronagraphy, $\varepsilon=2$. Prolate solutions to the coronagraphic equation exist for any set of eigenvalues satisfying the condition:

$\Lambda_{0 x} \Lambda_{0 y}=1 / 2$.

An infinite set of solutions exists, in the range $1 / 2<$ $\Lambda_{0 x}<1$ and $1 / 2<\Lambda_{0 y}<1$ (Fig. 1, top). The corresponding mask sizes are easily obtained as the inverse function of the eigenvalue curve, as illustrated in Fig. 1, bottom. These curves are generally drawn as a function of the socalled prolateness parameters $c_{x}$ and $c_{y}: c_{x}=\frac{\pi \alpha_{x}}{2}$ and $c_{y}=\frac{\pi \alpha_{y}}{2}$ (Papoulis 1981). This can be obtained by a proper change of variables in Eq. (8). For clarity, we have computed the eigenvalues as a function of the mask size $\left(\alpha_{x}\right.$ and $\left.\alpha_{y}\right)$, instead of the usual prolateness parameter.
Let us consider two possible sets of solutions, that we call $(A)$ and $(B)$. A first trivial solution $(A)$ is obtained for $\Lambda_{0 x}=\Lambda_{0 y}=\frac{1}{\sqrt{2}}$ (point $(A)$ of Fig. 1$)$. The corresponding mask size is $\alpha_{x}=\alpha_{y}=0.8476$ (expressed in resolution units $\lambda f(L)$. The transmissions in amplitude and intensity are illustrated in Fig. 2 for a rectangular aperture of size $L_{x}=4 L_{y}$.

This trivial solution is also the one with the highest transmission of the pupil. This can be shown as follows: let $T=T_{x} \times T_{y}$ be the energy transmission of the apodized rectangular aperture. Using Eq. (6), we have:

$T=\frac{1}{L_{x}} \int_{-L_{x} / 2}^{L_{x} / 2} \Phi_{x}^{2}(x) \mathrm{d} x \times \frac{1}{L_{y}} \int_{-L_{y} / 2}^{L_{y} / 2} \Phi_{y}^{2}(y) \mathrm{d} y$.

Using the relation giving the norm (Eq. (A.3)), we obtain:

$T=\frac{1}{L_{x}} \frac{\Lambda_{0 x}}{\Theta_{0 x}^{2}(0)} \times \frac{1}{L_{y}} \frac{\Lambda_{0 y}}{\Theta_{0 y}^{2}(0)}$.

and with Eq. (A.4):

$T=\frac{1}{4} \int_{-1}^{1} \frac{S_{00}^{2}\left(x, c_{x}\right)}{S_{00}^{2}\left(0, c_{x}\right)} \mathrm{d} x \times \int_{-1}^{1} \frac{S_{00}^{2}\left(y, c_{y}\right)}{S_{00}^{2}\left(0, c_{y}\right)} \mathrm{d} y$.

The functions $S_{00}$ depend on the prolateness parameters $c_{x}$ and $c_{y}$. However, $c_{x}$ and $c_{y}$ are independent of $L_{x}$ and $L_{y}$. The transmission $T$ is therefore independent of the rectangle proportions $L_{x} \times L_{y}$ for a given collecting area. A representation of the transmission as a function of $\Lambda_{0 x}$ is given in Fig. 3. The highest overall energy transmission of about $70.6 \%$ corresponds indeed to the trivial solution $\Lambda_{0 x}=\Lambda_{0 y}=0.707$.

However, other solutions may be of interest. A transmission corresponding to the solutions $\Lambda_{0 x}=0.505, \Lambda_{0 y}=$ 0.99 is drawn in Fig. 2. Note that the theoretical limit $\left(\Lambda_{0 x}=0.5, \Lambda_{0 y}=1\right)$ corresponds to an infinite mask slit of width $\alpha_{x}=0.540$. 


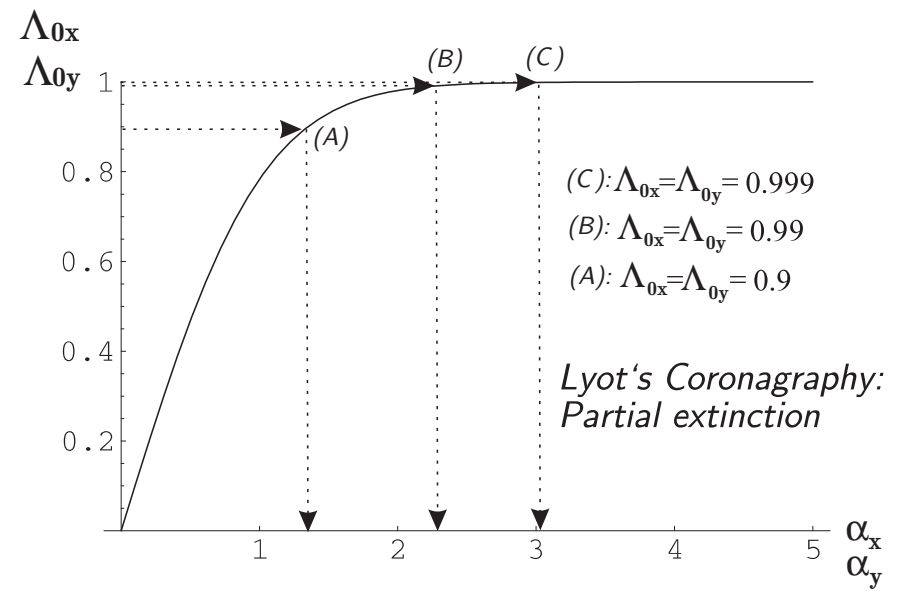

Fig. 4. For Lyot, the condition $\Lambda_{0 x} \times \Lambda_{0 y}=1$ cannot be obtained (it would correspond to an infinite mask size). However, approximate solutions exist, choosing eigenvalues close to 1 , as illustrated for the 3 configurations $(A),(B)$ and $(C)$ for eigenvalues close to 1 . The extinction is not total but the residue is optimal in terms of light concentration.

This permits one to reduce the mask size in one direction (and therefore reduces the dead zone in that direction). For a long slit aperture telescope (Touma et al. 1995, 1992; Martin et al. 1987), this is probably very interesting since it allows one to reduce the distance of planet detectability in the slit direction. In our example, $\alpha_{x}=0.547$ corresponds to a gain of $35 \%$ compared to solution $(A)$.

However, this gain in planet detectability is obtained to the detriment of the transmission for the planet (Fig. 3): in the example of $(B)$, the intensity transmission is only $47 \%$ compared to $70.6 \%$ for the $(A)$ setting.

It is interesting to represent the corresponding focal plane intensity for these apodized apertures, shown in Fig. 2 (right curves). Note that these figures also represent the PSF for planets situated well outside the coronagraphic mask, so that the extinction for the planet can be neglected.

\subsection{Residual amplitude for Lyot's coronagraphy $(\varepsilon=1)$}

There is no relevant solution to Eq. (7) for Lyot's coronography. Indeed, with $\varepsilon=1$, the exact solution is given by the condition $\Lambda_{0 x} \times \Lambda_{0 y}=1$ and the unique eigenvalue solutions $\Lambda_{0 x}=\Lambda_{0 y}=1$ would correspond to an infinite mask size (Fig. 4), i.e. an opaque mask occulting the whole field. However, approximate solutions can be obtained with a finite mask size. In that case, the extinction is only partial: the larger the mask, the lower the residual star light. However, prolate apodizations give surprising interesting results here too. Taking advantage of the rapid saturation of the eigenvalue after $\alpha_{x}=2$ (or $\alpha_{y}$ ) (Fig. 4), we can choose a mask size $\alpha_{x}$ (and $\alpha_{y}$ ) corresponding to eigenvalues close to 1 . A corresponding prolate apodization exists but it is no longer an exact solution of the coronagraphic equation.
The residual pupil plane amplitude $\Psi_{\mathrm{C}}(x, y)$ therefore can be written (subtituting Eq. (6) in Eq. (5)):

$$
\begin{aligned}
\Psi_{\mathrm{C}}(x, y)= & \Pi\left(\frac{x}{L_{x}}\right) \Pi\left(\frac{y}{L_{y}}\right)\left[\Pi\left(\frac{x}{L_{x}}\right) \Pi\left(\frac{y}{L_{y}}\right) \Phi(x, y)\right. \\
& -\Pi\left(\frac{x}{L_{x}}\right) \Phi_{x}(x) * \frac{\sin \pi\left(\alpha_{x} / L_{x}\right) x}{\pi x} \\
& \left.\times \Pi\left(\frac{y}{L_{y}}\right) \Phi_{y}(y) * \frac{\sin \pi\left(\alpha_{y} / L_{y}\right) y}{\pi y}\right]
\end{aligned}
$$

We express the two convolution products of Eq. (13) using Eq. (A.2) (and the two corresponding eigenvalues):

$$
\begin{aligned}
& \Pi\left(\frac{x}{L_{x}}\right) \Phi_{x}(x) * \frac{\sin \pi\left(\alpha_{x} / L_{x}\right) x}{\pi x}=\Lambda_{0 x} \Phi_{x}(x) \\
& \Pi\left(\frac{y}{L_{y}}\right) \Phi_{y}(y) * \frac{\sin \pi\left(\alpha_{y} / L_{y}\right) y}{\pi y}=\Lambda_{0 y} \Phi_{y}(y) .
\end{aligned}
$$

Combining Eq. (14) in Eq. (13), we obtain the surprising result:

$$
\begin{aligned}
\Psi_{\mathrm{C}}(x, y) & =\left[\left(1-\Lambda_{0 x} \Lambda_{0 y}\right)\right] \Phi_{x}(x) \Phi_{y}(y) \Pi\left(\frac{x}{L_{x}}\right) \Pi\left(\frac{y}{L_{y}}\right) \\
& =\left(1-\Lambda_{0 x} \Lambda_{0 y}\right) \Psi_{\mathrm{A}}(x, y) .
\end{aligned}
$$

The residual amplitude is itself proportional to the entrance amplitude. Examples of what can be obtained for a square aperture and different values of a square mask are shown in Fig. 6.

The integrated energy is simply $\left(1-\Lambda_{0 x} \Lambda_{0 y}\right)^{2}$ times the energy transmitted by the apodized aperture (Eq. (15)). The closer to 1 that the eigenvalues $\Lambda_{0 x}$ and $\Lambda_{0 y}$ lie, the better the coronagraphic rejection, but the larger the mask size (Fig. 4) and the stronger the apodization (loss of transmission and angular resolution). A tradeoff must be chosen between a small mask size and a high coronagraphic effect. Note that the mask size is always smaller than the core of the apodized PSF. This is one of the main differences to classical Lyot's coronagraphy for which the mask extends over several Airy rings (Fig. 6).

The residual integrated energy of the star decreases very rapidly (roughly exponentially) as a function of the mask size. This result may be useful to determine the tradeoff between the star light rejection and the mask size for Lyot's coronagraphy, as illustrated in Fig. 6.

Equation (15) shows that the residual amplitude inside the aperture is itself proportional to the entrance prolate apodization. The residual wave amplitude in the exit pupil is therefore apodized, and is simply proportional to the entrance pupil amplitude, to the factor $\left(1-\Lambda_{0 x} \Lambda_{0 y}\right)$ : the overall effect of Lyot's coronagraphy is simply to reduce the intensity of the star in the final focal plane, with the same intensity pattern as for the planet. This is a very surprising result: it is generally assumed that most of the residual star light appears at the edges of the pupil for classical Lyot's coronagraphy, without apodization.

Moreover, the prolate function is also the optimal apodization in terms of light concentration for the star residual light: it maximizes the integrated intensity within the coronagraphic masks (more comments in 
Normalized residual energy of the star

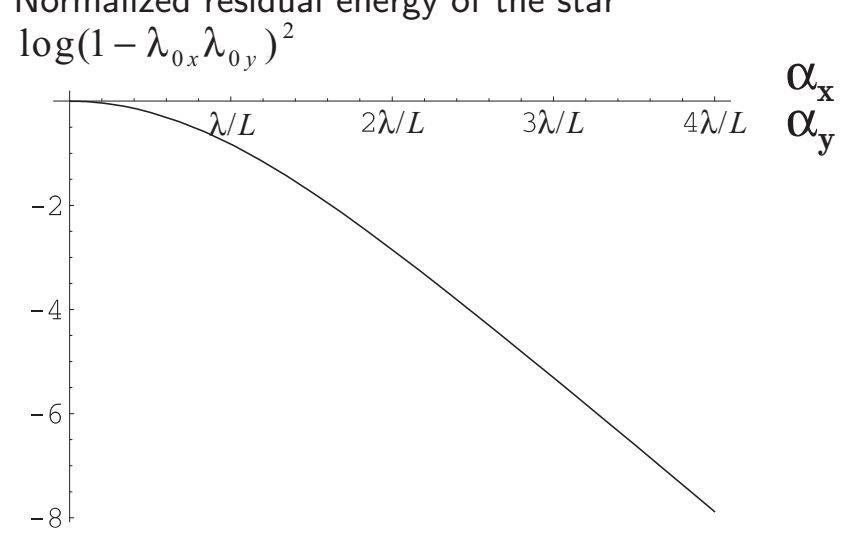

Pupil energy transmission

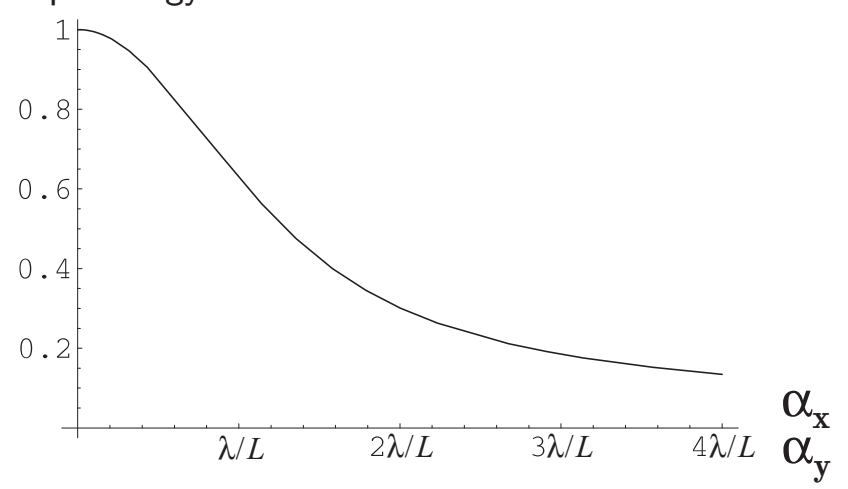

Angular resolution

(first zero of the apodized PSF)

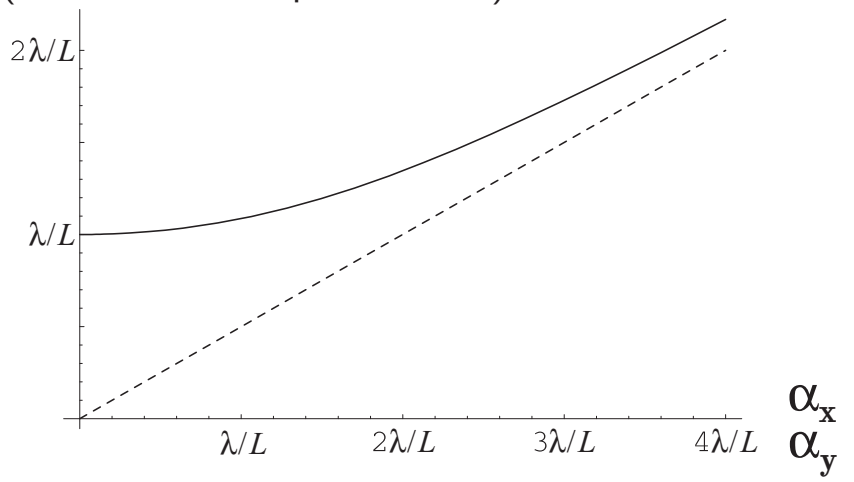

Fig. 5. Results for Lyot's coronagraphy with increasing accuracy of the approximate solution (increasing eigenvalues, apodization strength and mask size, see also Fig. 6), as a function of the corresponding mask size. Here, the mask is such that $\alpha_{x}=\alpha_{y}$. Top: residual energy for the star, normalized to the entrance pupil transmission. Center: illustration of the loss of transmission for the approximate solutions due to the increasing strength of the apodization. Bottom: illustration of the loss of resolution due to the apodization. The full line represents the position of the first zero of the PSF and can be compared to the half width of the mask (dotted line).

Appendix A). We cannot obtain a total star light extinction for Lyot's coronagraphy, but with prolate apodization, the residue is optimum. Using Eq. (A.1), we can obtain an analytical expression for the residual amplitude of the star in the final focal plane for Lyot's coronagraphy:

$$
\begin{aligned}
\Psi_{\mathrm{D}}(x, y)= & \left(1-\Lambda_{0 x} \Lambda_{0 y}\right) L_{x} L_{y} \sqrt{\frac{\Lambda_{0 x}}{\alpha_{x}}} \sqrt{\frac{\Lambda_{0 y}}{\alpha_{y}}} \\
& \times \Phi_{x}\left(\frac{L_{x}^{2}}{\alpha_{x}} \frac{x}{\lambda f}\right) \Phi_{y}\left(\frac{L_{y}^{2}}{\alpha_{y}} \frac{y}{\lambda f}\right) .
\end{aligned}
$$

Examples of the residual focal intensity are given in Fig. 6 for the 3 configurations, corresponding to rejection rates of $3.6 \times 10^{-2}, 4.0 \times 10^{-4}, 4.0 \times 10^{-6}$. Very high rejections can be theoretically obtained with Lyot's coronagraphy and prolate apodization, to the detriment of the transmission and the angular resolution.

\section{Alternative presentation of the particular interest of prolate functions for coronagraphy}

In the previous sections, we used the property of linear prolate functions, relative to their invariance to the sine cardinal kernel: we have seen (Eq. (8)) that the central part of a prolate function convolved by a sine cardinal function is similar to the same (unbounded) prolate function. Now we examine this property in Fourier space. The Fourier Transform (FT) of the central part of a prolate function is similar to the same unbounded prolate function (see Appendix A), and reciprocally. It has been shown that prolate functions are the only functions that enjoy this property (Slepian \& Pollak 1961; Frieden 1971).

This property is fundamental for coronagraphy, in which several successive FT are involved from the entrance pupil plane to the final observing plane, and where the complex amplitude of the wave is successively bounded by different masks and diaphragms.

Let us first examine the example of Roddier \& Roddier's coronagraphy. For simplicity, we present here the one-dimensional case.

Figure 7 describes the wave amplitude at the successive planes A, B and C. We assert that the same prolate function appear in all these planes (to a scaling factor).

At the pupil plane $\mathrm{A}$, the telescope transmission, between $-L_{x} / 2$ and $L_{x} / 2$, is proportional to the central part of a prolate function. This function is represented (with a dashed line) outside the telescope aperture. The FT of the aperture transmission (Goodman 1996) gives in the focal plane an amplitude response which is exactly similar to the prolate function of plane A:

$$
\begin{aligned}
\Psi_{\mathrm{B}}(x) & =\int_{-L_{x} / 2}^{L_{x} / 2} \Phi_{x}(t) \mathrm{e}^{2 \imath \pi \frac{x}{\lambda f}} \mathrm{~d} t \\
& =L_{x} \sqrt{\frac{\Lambda_{0 x}}{\alpha_{x}}} \Phi_{x}\left(\frac{L_{x}^{2}}{\alpha_{x}} \frac{x}{\lambda f}\right) \\
& =L_{x} \sqrt{\frac{\Lambda_{0 x}}{\alpha_{x}}} \Phi_{x}\left(\frac{x L_{x}}{m_{x}}\right) .
\end{aligned}
$$

In plane $\mathrm{B}$, the $\pi$ phase mask inverts the complex amplitude over a central part of the wave. In Eq. (17), the wave 

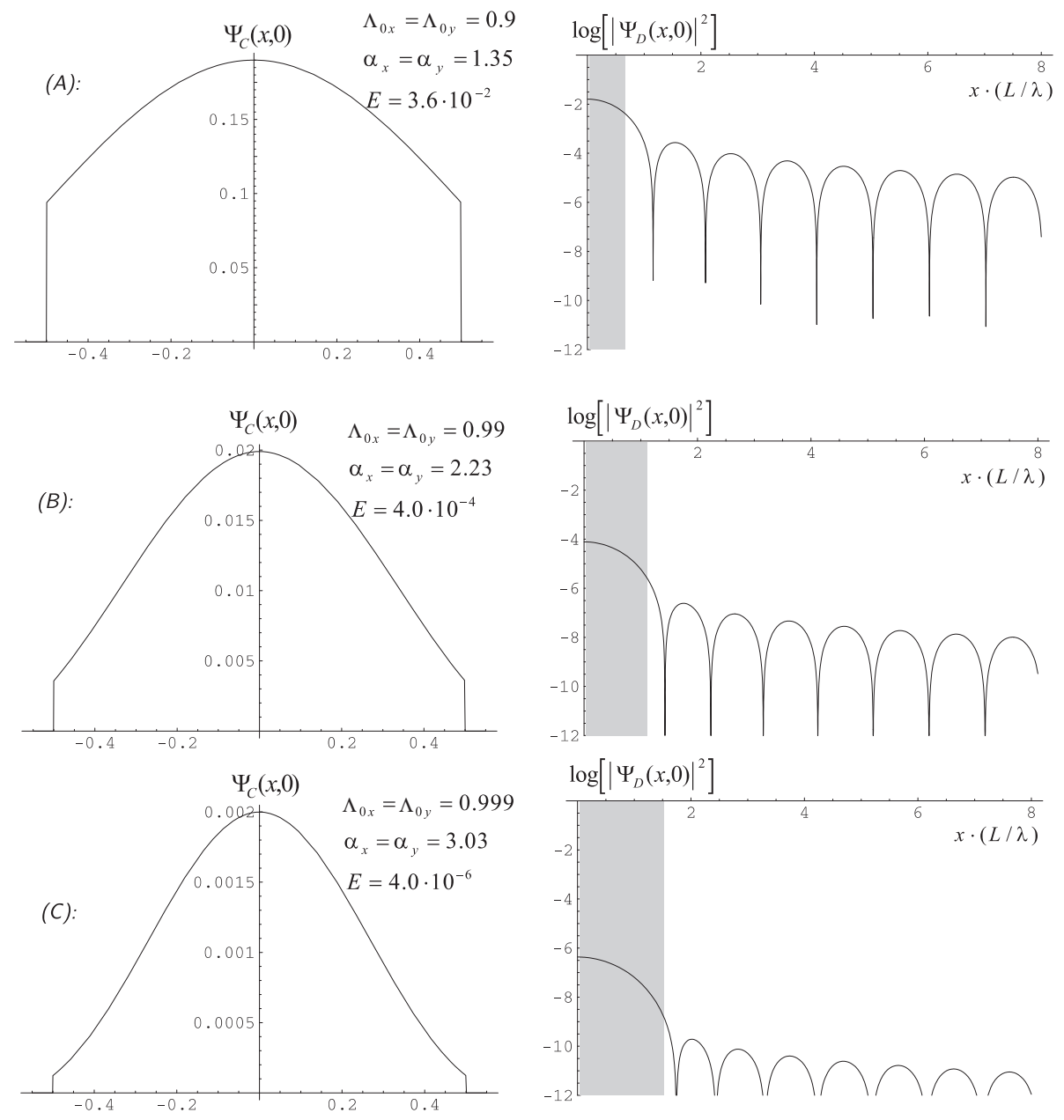

Fig. 6. Left: Slice of the entrance apodized pupil for Lyot's coronagraphy for the 3 configurations $(A),(B),(C)$ given in Fig. 4. The mask sizes are given in units of resolution, and we give the integrated residual star energy (normalized to the pupil transmission). Right: Slices of the residual star intensity in the final focal plane. Because of the prolate properties, these intensities are not dark at the center. The grey-tint rectangles show the corresponding width of the mask for each configuration.

amplitude inside the mask (for $-m_{x} / 2 \leq x \leq m_{x} / 2$ ) is simply homothetic to the apodized entrance pupil (shown in grey tint in Fig. 7).

If the focal plane is free of any coronagraphic mask (dashed line in plane B), a perfect image of the aperture is obtained in plane $\mathrm{C}$ (grey tint, top image in plane $\mathrm{C}$ ). The amplitude inside the mask gives, by diffraction from plane $\mathrm{B}$ to plane $\mathrm{C}$, an amplitude that is the exact negative reproduction (unbounded) of the entrance apodizing function (grey tint, bottom image in plane C). The coherent addition of these two amplitudes (the pupil and the coronagraphic term, i.e. the convolution product of Eq. (3)) is thus null between $-L_{x} / 2$ and $L_{x} / 2$ : the coronagraphy is perfect for an axial monochromatic point source:

$$
\begin{aligned}
\Psi_{\mathrm{C}}(x)= & \Phi_{x}(x) \mathrm{P}(x) \\
& -\frac{2}{\lambda f} \int_{-m_{x} / 2}^{m_{x} / 2} L_{x} \sqrt{\frac{\Lambda_{0 x}}{\alpha_{x}}} \Phi_{x}\left(\frac{t L_{x}}{m_{x}}\right) \mathrm{e}^{2 \imath \pi \frac{x}{\lambda f} t} \mathrm{~d} t .
\end{aligned}
$$

Performing a change of variables, we obtain:

$$
\begin{aligned}
\Psi_{\mathrm{C}}(x)= & \Phi_{x}(x) \mathrm{P}(x) \\
& -2 \frac{\alpha_{x}}{L_{x}^{2}} L_{x} \sqrt{\frac{\Lambda_{0 x}}{\alpha_{x}}} \int_{-L_{x} / 2}^{L_{x} / 2} \Phi_{x}(t) \mathrm{e}^{2 \imath \pi \frac{\alpha_{x}}{L_{x}} x t} \mathrm{~d} t \\
\Psi_{\mathrm{C}}(x)= & \Phi_{x}(x) \mathrm{P}(x)-2 \Lambda_{0 x} \Phi_{x}(x) .
\end{aligned}
$$

A Lyot's stop is necessary to remove the light diffracted outside the telescope aperture. It can be moreover verified that all the energy is rejected outside the aperture.

The same considerations can be made for Lyot's coronagraphy, although the extinction will not be total. The main difference comes from the fact that the Lyot's mask only sets to zero the central part of the wave. With eigenvalues close to 1 , we can obtain an approximate solution with interesting properties: the coronagraphic term is proportional to the pupil, by the factor $\Lambda_{0 x} \Lambda_{0 y}$, and the residual amplitude is also proportional to the pupil amplitude, by the factor $\left(1-\Lambda_{0 x} \Lambda_{0 y}\right)$. The response for an on-axis point source is thus identical and reduced in intensity.

\section{Conclusion and discussion}

In this paper we have introduced a theoretical formalism for coronagraphy of rectangular apertures with entrance pupil apodization: we have shown that the natural apodization functions for Lyot's coronagraphy and Roddier \& Roddier's coronagraphy are the linear prolate spheroidal functions.

We have proven that a total extinction of a monochromatic unresolved on-axis star can be obtained using a rectangular aperture apodized by prolate functions (in $x$ and $y$ ) and Roddier \& Roddier's phase mask 


\section{Roddier \& Roddier's Coronagraphy}

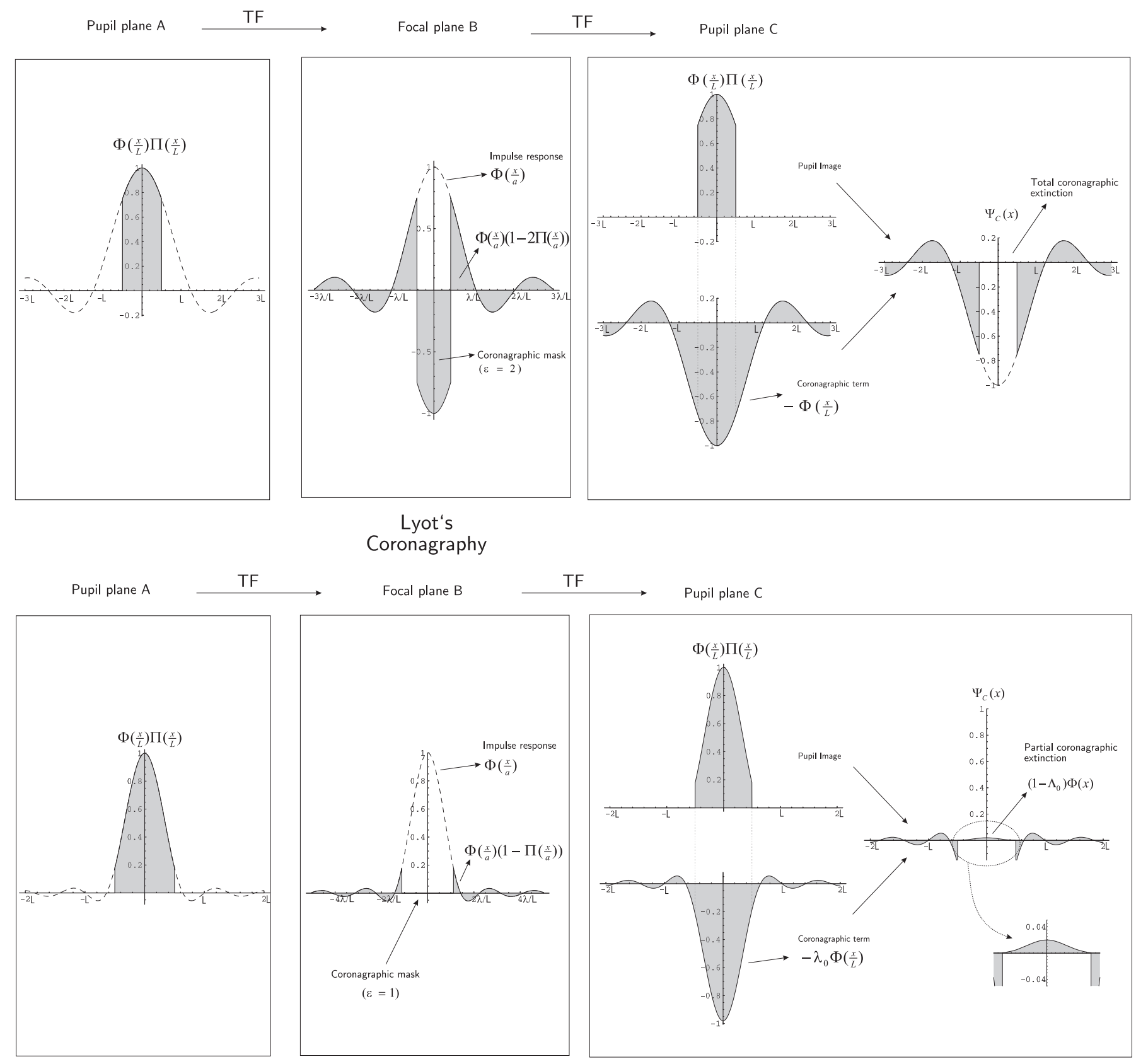

Fig. 7. Illustration of the interest of prolate apodizations for coronagraphy. Plane A: telescope aperture apodized using a linear prolate. Plane B: the wave inside the mask for R\&R (or occulted by the mask for Lyot) is a scaled image of the entrance pupil. Plane C: the pupil and the coronagraphic term are equal inside the aperture for $R \& R$, and proportional for Lyot. The residual amplitude is null for R\&R, and proportional to the entrance pupil amplitude for Lyot.

coronagraphy. Several pupil apodizations can give this result: within a range of values, one can define the prolate apodization (and corresponding mask size) to use lengthwise, and the prolate apodization in the other direction is then fixed. The best intensity transmission is obtained when the two apodizations are equal (in terms of normalized dimensions of the rectangle). It might be interesting to increase the resolution in one direction (using a smaller mask size in that direction), with the drawback of a lower resolution in the other direction and a lower overall aperture transmission.

The study we have made is for a monochromatic wave front. R\&R's coronagraphy is said to be more sensitive to wavelength effects than other phase shifting techniques, such as the wavelength-independent CIA (Gay \& Rabbia 1996; Baudoz et al. 2000a,b) or the less sensitive 4QC 
technique (Rouan et al. 2000), or the achromatic PKC (Abe et al. 2001, 2002). To overcome this problem, one can use an achromatization system that produces a magnification of the focal image proportional to 1 / $\lambda$ over the bandpass (Wynne 1979; Roddier et al. 1980). Then the aperture image must undergo the same procedure (in the reverse sense) for the Lyot stop.

For the original Lyot's technique, with the same technique, only a partial extinction of the star can be obtained, but there also, prolate apodizations give optimal results. Both the star and the planet appear in the final image with the same Point Spread Function (PSF) that is optimal in terms of concentration of light, because of the prolate properties. The overall effect of Lyot's coronagraphy with prolate apodization is then to reduce the magnitude difference between the planet and its parent star. The reduction factor obtained with the prolate apodization is good enough to consider Lyot's technique as a valid challenger of Roddier \& Roddier's technique, mainly because of its simplicity and its lower dependence on the deviation from the perfect case: chromatism, star diameter, telescope jitter (Aime et al. 2001a). The most interesting result with Lyot's coronagraphy and prolate apodization is probably the fact that the residual wavefront on the aperture for the starlight is again a prolate apodized aperture. Then it seems conceivable to undertake a second Lyot's coronagraphy. If this two-step (or even more!) experiment appears to be feasible, then Lyot's coronagraphy with a prolate apodized aperture will permit one to obtain considerable reduction factors for a reasonable mask size. This is an aspect we intend to study in a future work. One of the difficulties in any case will be the realization of the prolate apodizer. These functions are indeed very close to a cosine squared arch, as we show in Appendix B. This transmission can be realized by an interference process (Aime et al. 2001a). However, cosine apodizations do not enjoy the very interesting properties of prolate functions described above.

This paper simply intended to present a theoretical and analytical approach for coronagraphy in space using apodized apertures. Several issues have not been tackled in this paper and remain to be studied numerically. In particular, numerical simulations will be needed to evaluate the sensitivity of these formal solutions to the physical parameters: wavelength dependence, wavefront errors, mask positioning errors, angular diameter of the star, etc. It will be also interesting to check if the technique can give valuable results from the ground using an apodized aperture with adaptive optics.

The technique has been described for a rectangular aperture: the interest of linear or rectangular apertures for exoplanet direct detection has already been discussed (Aime et al. 2001a,b): they provide a very favorable configuration to overcome the chromatism limitations, using a $X, \lambda$ coronographic mode.

However, similar results could be obtained for a full circular aperture (with no central obstruction) substituting the circular prolate function for the linear one
(Frieden 1971). This will be described in a future work. The use of prolate apodization for diluted apertures is a much more difficult problem for which no theoretical approach seems to exist yet.

Acknowledgements. The authors would like to thank Paul Abbott and Peter Falloon for providing us the Mathematica Package for the computing of the prolate functions. Thanks are also due to F. Malbet and D. Mouillet, for their careful reading of the manuscript and constructive comments.

\section{Appendix A: Linear prolate functions}

As well described by Frieden (1971), prolate functions, known since Niven (1880), were re-discovered by Splepian in 1959 to find the bandlimited function that is maximally concentrated, in the mean square sense, within a given time interval. As shown in Sect. 4, the transposition to Optics is immediate, identifying the aperture with the bandlimited spectrum, and the focal plane with the time domain. This uses classical properties of Fourier Optics (Goodman 1996). The mean square consideration for the amplitude means that the prolate apodization maximizes the integrated intensity within a given region of the focal plane, here the coronagraphic mask size. This property may be used for itself if apodization alone was to be considered (Nisenson \& Papaliolios 2001).

The first publication on prolate functions in signal processing is by Slepian \& Pollak (1961). See also the very comprehensive presentation of Papoulis (1981).

Prolate functions have found very interesting applications in Optics. The short presentation we give here is drawn from Frieden (1971). Notations and parameters are modified to fit the present application.

As well emphasized by Frieden (1971), linear prolate functions are defined by their invariance to a finite Fourier Transform:

$\int_{-L_{x} / 2}^{L_{x} / 2} \Theta_{n}(t) \mathrm{e}^{2 \imath \pi \nu t} \mathrm{~d} t=\imath^{n} L_{x} \sqrt{\frac{\Lambda_{n}}{\alpha_{x}}} \Theta_{n}\left(\frac{\nu L_{x}^{2}}{\alpha_{x}}\right)$.

Performing a finite Fourier transform on both sides of Eq. (A.1), we have:

$\int_{-L_{x} / 2}^{L_{x} / 2} \Theta_{n}(\xi) \frac{\sin \pi\left(\alpha_{x} / L_{x}\right)(x-\xi)}{\pi(x-\xi)} \mathrm{d} \xi=\Lambda_{n} \Theta_{n}(x)$

(the finite FT of a function is the FT of the truncated function).

For the apodization problem we consider, as in many optical problems, that only the first prolate spheroidal function $\Theta_{0}(x)$ is of practical interest since it is the only one that is maximum at the origin (Frieden 1971). We easily recognize here a part of the coronagraphic equation for rectangular apertures (Eq. (8)), with $\Lambda_{0 x}=\frac{1}{\varepsilon_{x}}$. This integral equation admits as solutions (eigenfunctions) the linear prolate spheroidal functions $\Theta_{0}(x)$ with eigenvalues $\Lambda_{0}$. 


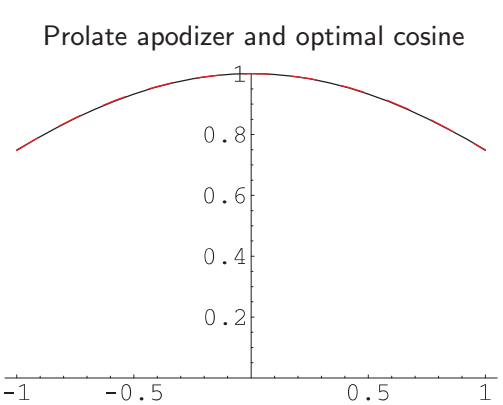

Prolate apodizer and optimal $\cos ^{2}$

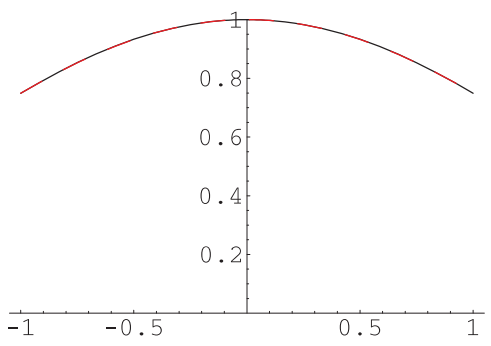

\begin{abstract}
Relative difference
\end{abstract}

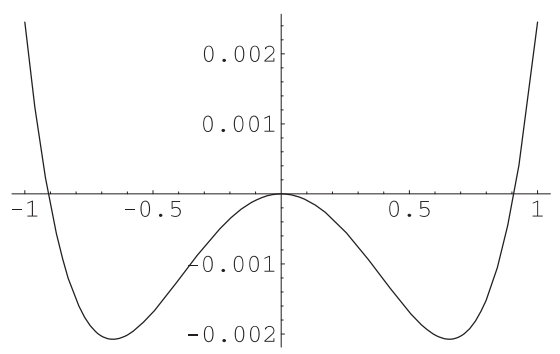

Relative difference

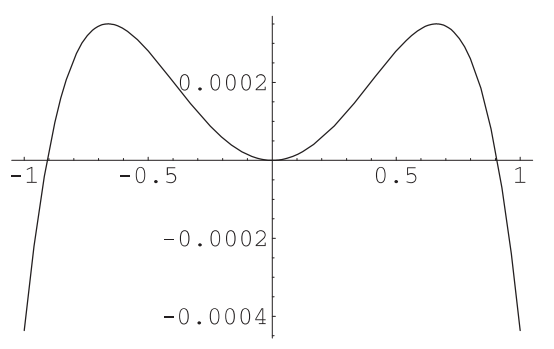

Fig. B.1. Comparison between optimal cosine and cosine squared apodization for Roddier \& Roddier's coronagraphy, with the exact prolate solution that permits a total extinction. Left: Amplitude aperture transmissions. At this scale, there is no difference between the prolate function and the cosine (top) or cosine squared (bottom) apodizations of Aime et al. (2001a). Right: residual amplitude in plane $\mathrm{C}$ in favor to the cosine squared compared to the the simple cosine apodization.
The prolate functions have an interesting double orthogonality property:

$\int_{-L_{x} / 2}^{L_{x} / 2} \mathrm{~d} x \Theta_{n}(x) \Theta_{m}(x)=\Lambda_{n} \delta_{m n}$
$\int_{-\infty}^{\infty} \mathrm{d} x \Theta_{n}(x) \Theta_{m}(x)=\delta_{m n}$

where $\delta_{m n}$ is the Kronecker delta.

The linear prolate functions can be computed from the angular prolate spheroidal functions which are the solutions to the wave equation propagation in prolate spheroidal coordinates (Flammer 1957; Abramowitz \& Stegun 1970; Abbott 1997):

$\Theta_{n}(x)=\sqrt{\frac{2 \Lambda_{n x}}{L_{x}}} \mathrm{~S}_{0 n}\left(2 x / L_{x}, c_{x}\right)\left(\int_{-1}^{1} \mathrm{~S}_{0 n}^{2}\left(t, c_{x}\right) \mathrm{d} t\right)^{-1 / 2}$.

We used the Mathematica package (Wolfram 1999) to compute the linear prolate functions and the eigenvalues. This package was written and kindly given to us by Falloon (2001).

\section{Appendix B: Cosine or cosine squared apodizations as approximations of prolate apodizations}

In a recent paper (Aime et al. 2001a), we proposed an interferometric apodization technique which is able to produce apodization, in the shape of a cosine, or any power of a cosine, and studied its application to coronagraphy. Cosine and cosine squared apodizations turned out to be extremely efficient solutions for Lyot's and Phase Mask coronagraphy, provided that a partial central arch was used. The apodization function was of the form:

$P_{X}^{N}(x)=\cos ^{N}\left(\frac{\pi x}{b L_{x}}\right) \times \Pi\left(\frac{x}{L_{x}}\right)$,

with $N=1$ and $N=2$. The optimal parameter $b$ is obtained minimizing the residual energy left by the coronagraph for an unresolved monochromatic point source. The optimal cosine and cosine squared functions are actually extremely close to the exact prolate solution, as shown in Fig. B.1. This explains their excellent efficiency for PM coronagraphy.

Moreover, the mask size $\alpha_{x}=0.847586$ for the prolate solution (associated to the eigenvalue $\Lambda_{0}=\frac{1}{\sqrt{2}}$ ) is very close to the optimal mask size obtained for cosine apodizations. The relative difference between them is only $8.7 \times 10^{-6}$ for cosine apodization and $7.7 \times 10^{-7}$ for cosine squared apodization.

For Lyot's coronagraphy, the comparison is not so easy since no exact prolate solution exists. We have chosen to use in this comparison the approximated prolate solution closest to the optimal cosine or cosine squared (i.e. this imposes a point in Fig. 4).

The results are illustrated in Fig. B.2. Again, we notice that a much better fit of a prolate is given by the cosine squared. The prolate function corresponding to the cosine squared is associated with a higher eigenvalue $\Lambda_{0}=0.997$ than the prolate corresponding to the cosine $\left(\Lambda_{0}=0.984\right)$ and thus leads to a lower residual energy. $\left(6.67 \times 10^{-4}\right.$ for cosine apodization and $2.95 \times 10^{-5}$ for cosine squared apodization and a square aperture). The overall intensity transmission of the apodizers are respectively: $30 \%$ and $22 \%$. 

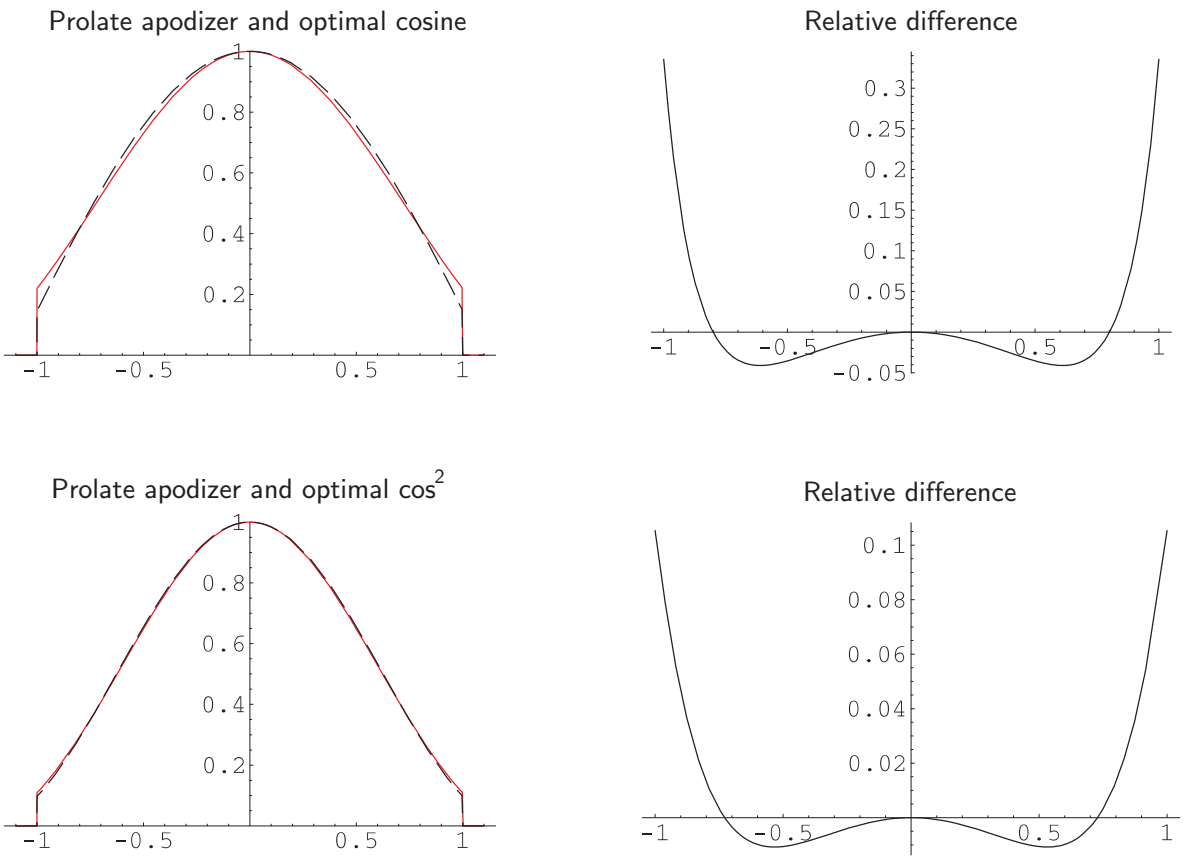

Fig. B.2. Comparison between optimal cosine and cosine squared apodization for Lyot's coronagraphy, with the closest approximated prolate solution. Left: Amplitude aperture transmission. The cosine apodization (dashed line) hardly fits the prolate shape $\left(\Lambda_{0}=\right.$ 0.984 . The agreement is better between cosine squared and prolate $\left(\Lambda_{0}=\right.$ 0.997). Right: residual amplitude in plane C. Cosine apodizations do not have the remarkable properties of the prolate ones. The star light is rejected on the edges of the aperture.

\section{References}

Abbott, P. 1997, Math. J., 7.1

Abe, L., Domiciano de Souza, J., Vakili, F., \& Gay, J. 2002, A\&A, to be published

Abe, L., Vakili, F., \& Boccaletti, A. 2001, A\&A, 374, 1161

Abramowitz, M., \& Stegun, I. A. 1970, Handbook of mathematical functions

Aime, C., Soummer, R., \& Ferrari, A. 2001a, A\&A, 379, 697

Aime, C., Soummer, R., \& Lopez, B. 2001b, A\&A, 370, 680

Baudoz, P. 1999, Ph.D. Thesis, Observatoire de la Côte d'Azur, Nice, France

Baudoz, P., Rabbia, Y., \& Gay, J. 2000a, A\&AS, 141, 319

Baudoz, P., Rabbia, Y., Gay, J., et al. 2000b, A\&AS, 145, 341

Beuzit, J.-L., Mouillet, D., Lagrange, A.-M., \& Paufique, J. 1997, A\&AS, 125, 175

Bonneau, D., Josse, M., \& Labeyrie, A. 1975, in ASSL Vol. 54: Image Processing Techniques in Astronomy, 403

Falloon, P. 2001, Master's Thesis, University of Western Australia

Flammer, C. 1957, Spheroidal Wave Functions, ed. S. U. Press

Frieden, B. R. 1971, Progress in Optics, ed. E. Wolf, 9

Gay, J., \& Rabbia, Y. 1996, C. R. Acad. Sci Paris, 322 Sér. II b, 265

Goodman, J. 1996, Introduction to Fourier Optics, ed. M. G. Hill

Guyon, O., \& Roddier, F. 2000, in Proc. of SPIE, 40006

Lyot, B. 1930, C. R. Acad. Sci Paris, 191, 834
Lyot, B. 1939, MNRAS, 99, 580

Malbet, F. 1996, A\&AS, 115, 161

Martin, F., Touma, H., Bijaoui, A., \& Aime, C. 1987, J. Opt., 18,133

Mouillet, D. 1997, Ph.D. Thesis, Laboratoire d'Astrophysique de l'Observatoire de Grenoble, France

Mouillet, D., Lagrange, A.-M., Beuzit, J.-L., \& Renaud, N. 1997, A\&A, 324, 1083

Nisenson, P., \& Papaliolios, C. 2001, ApJL, 549, 201

Niven, C. 1880, Phil. Trans. Roy. Soc. London, Ser. A 171, 117

Papoulis, A. 1981, Signal Analysis, ed. M. G. Hill

Roddier, C., Roddier, F., Martin, F., Baranne, A., \& Brun, R. 1980, J. Opt., 11, 149

Roddier, F., \& Roddier, C. 1997, PASP, 109, 815

Rouan, D., Riaud, P., Boccaletti, A., Clénet, Y., \& Labeyrie, A. 2000, PASP, 112, 1479

Slepian, D., \& Pollak, H. O. 1961, Bell Sys. Tech. J., 40, 43

Smith, B. A., \& Terrile, R. J. 1984, Science, 226, 1421

Touma, H., Martin, F., \& Aime, C. 1995, Pure Appl. Opt., 4, 685

Touma, H., Martin, F., Kadiri, S., \& Aime, C. 1992, in Progress in Telescope and Instrumentation Technologies, 527

Watson, S. M., Mills, J. P., Gaiser, S. L., \& Diner, D. J. 1991, Appl. Opt., 30, 3253

Wolfram, S. 1999, The Mathematica book, Fourth Edition (Cambridge University Press)

Wynne, C. G. 1979, Opt. Commun., 28, 21 